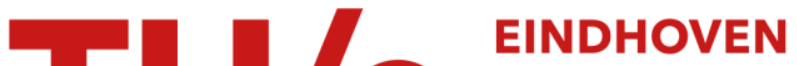

\section{The effect of global and local damping on the perception of hardness}

Citation for published version (APA):

van Beek, F., Heck, D., Nijmeijer, H., Bergman Tiest, W. M., \& Kappers, A. (2016). The effect of global and local damping on the perception of hardness. IEEE Transactions on Haptics, 9(3), 409-420.

https://doi.org/10.1109/TOH.2016.2567395

\section{Document license:}

TAVERNE

DOI:

10.1109/TOH.2016.2567395

Document status and date:

Published: 01/07/2016

\section{Document Version:}

Publisher's PDF, also known as Version of Record (includes final page, issue and volume numbers)

\section{Please check the document version of this publication:}

- A submitted manuscript is the version of the article upon submission and before peer-review. There can be important differences between the submitted version and the official published version of record. People interested in the research are advised to contact the author for the final version of the publication, or visit the $\mathrm{DOI}$ to the publisher's website.

- The final author version and the galley proof are versions of the publication after peer review.

- The final published version features the final layout of the paper including the volume, issue and page numbers.

Link to publication

\section{General rights}

Copyright and moral rights for the publications made accessible in the public portal are retained by the authors and/or other copyright owners and it is a condition of accessing publications that users recognise and abide by the legal requirements associated with these rights.

- Users may download and print one copy of any publication from the public portal for the purpose of private study or research.

- You may not further distribute the material or use it for any profit-making activity or commercial gain

- You may freely distribute the URL identifying the publication in the public portal.

If the publication is distributed under the terms of Article 25fa of the Dutch Copyright Act, indicated by the "Taverne" license above, please follow below link for the End User Agreement:

www.tue.nl/taverne

Take down policy

If you believe that this document breaches copyright please contact us at:

openaccess@tue.nl

providing details and we will investigate your claim. 


\title{
The Effect of Global and Local Damping on the Perception of Hardness
}

\author{
Femke E. van Beek, Dennis J.F. Heck, Henk Nijmeijer, Fellow, IEEE, \\ Wouter M. Bergmann Tiest, and Astrid M.L. Kappers
}

\begin{abstract}
In tele-operation systems, damping is often injected to guarantee system stability during contact with hard objects. In this study, we used psychophysical experiments to assess the effect of adding damping on the user's perception of object hardness. In Experiments 1 and 2, combinations of stiffness and damping were tested to assess their effect on perceived hardness. In both experiments, two tasks were used: an in-contact task, starting at the object's surface, and a contact-transition task, including a free-air movement. In Experiment 3, the difference between inserting damping globally (equally throughout the workspace) and locally (inside the object only) was tested. In all experiments, the correlation between the participant's perceptual decision and force and position data was also investigated. Experiments 1 and 2 show that when injecting damping globally, perceived hardness slightly increased for an incontact task, while it decreased considerably for a contact-transition task. Experiment 3 shows that this effect was mainly due to inserting damping globally, since there was a large perceptual difference between inserting damping globally and locally. The force and position parameters suggest that participants used the same force profile during the two movements of one trial and assessed the system's reaction to this force to perceive hardness.
\end{abstract}

Index Terms-Haptics, psychophysics, damping, hardness, stiffness, tele-operation

\section{INTRODUCTION}

$\mathrm{N}$ EW technologies like deep-sea mining, remote handling for nuclear technologies and space applications rely heavily on the use of tele-operation systems. In these systems, the operator uses a master device to control a slave system in the remote environment. By establishing a feedback loop from the remote environment to the operator, a bilateral connection is created and the operator can be provided with force feedback from the remote environment. If the quality of the feedback is very good, which typically implies that the impedance of the remote environment is reflected properly through the force feedback signal, the operator is provided with a very accurate representation of the remote environment [1]. An important factor determining the feedback quality is the choice of the control architecture governing the bilateral connection, which depends on several system properties.

One of those properties is the presence of delays in the system [2]. If the master and slave are physically separated over a large distance, there is a delay between sending information at one end and receiving it at the other end. These delays can cause instabilities in the system, which is an undesirable property, since they can damage the teleoperator or

- F.E. van Beek, W.M.B. Tiest and A.M.L. Kappers are with the MOVE Research Institute Amsterdam, Department of Human Movement Sciences, Vrije Universiteit Amsterdam, Amsterdam, The Netherlands. E-mail:\{f.e.van.beek,w.m.bergmanntiest, a.m.l.kappers\}@vu.nl.

- D.J.F. Heck and H. Nijmeijer are with the Dynamics and Control group, Department of Mechanical Engineering, Eindhoven University of Technology, Eindhoven, The Netherlands. E-mail: \{D.J.F.Heck, h.nijmeijer\}@tue.nl.

Manuscript received 7 Dec. 2015; revised 24 Apr. 2016; accepted 8 May 2016. Date of publication 12 May 2016; date of current version 14 Sept. 2016.

Recommended for acceptance by K. Drewing.

For information on obtaining reprints of this article, please send e-mail to: reprints@ieee.org, and reference the Digital Object Identifier below.

Digital Object Identifier no. 10.1109/TOH.2016.2567395 environment and prevent the operator from executing the task. Nuño et al. [3] have shown that one way to guarantee stability is to ensure that the ratio of injected damping by the controller and the proportional controller gain (which is strongly related to the stiffness reflected to the operator) is bounded from below. This bound, and thus the required amount of damping, increases for increasing delays. Hence, a common solution to prevent instabilities is to inject of a lot of damping or viscous friction into the system through the controller (see e.g., [4], [5], [6]). In this way, the stability is guaranteed in the presence of delays and, at least theoretically, it remains possible to reflect high stiffnesses, which would make the device potentially capable of providing high quality feedback. However, it is unclear what the effect of injecting damping is on the operator's perception of the remote environment. One of the important aspects conveyed by high quality haptic feedback is object hardness, since the proper assessment of hardness is crucial to identify objects and to properly interact with them. A surgeon, for instance, needs to feel the difference in hardness between the tumor that must be removed and the surrounding tissue that needs to stay intact. Moreover, the proper experience of hardness allows operators to exert the right amount of force to manipulate an object without damaging it. Therefore, the question in our study was: what is the effect of injecting damping on the perception of object hardness?

The perception of hardness of natural objects has been investigated quite thoroughly (see Bergmann Tiest [7] for a review), which has shown that stiffness plays a major role in making objects feel hard. The relationship between stiffness and perceived hardness of natural objects can be described by a power function with an exponent of $\sim 0.8$ [8], while the Just Noticeable Difference (JND) for stiffness is $\sim 15$ percent [9], [10]. For virtual objects, Lawrence et al. [11] 
have shown that force rate of change at impact divided by displacement velocity at impact seems to correlate better with perceived hardness than stiffness alone does. They called this new parameter 'rate-hardness'. Han and Choi [12] extended this work by describing a slightly different parameter, 'extended rate hardness', which they defined as maximum force rate of change divided by displacement velocity at impact, which should be suitable for a larger class of rendering algorithms. Lawrence et al. [11] stated that rate-hardness can be seen as a sum of stiffness and damping, leading to the final rate-hardness value. This underlines the potential importance of damping in the perception of hardness.

Rosenberg and Adelstein [13] have shown that directional dampers can provide a sensation of hardness and can feel 'wall-like', which suggests that damping can contribute to the perceived hardness of an object. However, the authors did not combine damping and stiffness in one object. Lawrence et al. [11] did use a control law to quickly adjust the object's stiffness, which should have resulted in something that resembles a combination of stiffness and damping. When limiting the interaction force to $2 \mathrm{~N}$, they found that adding a damping-like parameter through this control law increased the perceived object hardness in a free exploration task. Han and Choi [12] essentially replicated the findings in a similar experiment using a comparable type of control law, while using a much stronger haptic device (maximum force $37.5 \mathrm{~N}$ ) and a tapping task. However, in both studies the amount of damping was not controlled directly and therefore it is not known which amount of damping corresponds to which amount of increase in perceived hardness. Moreover, both studies inserted damping inside the object only (which we call 'local damping'), while the injection of damping in control architectures for delayed bilateral teleoperation is typically done throughout the complete workspace, resulting in the same damping value both inside virtual objects and in free-space. So, the effects of the latter type of damping (which we call 'global damping') on the perception of hardness is still an open question.

In this study, we investigated the effect of damping on the perceived hardness of a virtual object, composed of a spring. The design mimics a common solution to guarantee stability in bilateral teleoperation, which is the injection of the same amount of damping inside objects and in freespace ('global damping'), and is therefore interesting for control engineers who aim to design high performance architectures. In Experiment 1, we studied the effect of injecting damping globally on the perception of hardness for various levels of stiffness. The results of Experiment 1 have been published previously in conference proceedings [14]. In Experiment 2, we studied the effect of injecting damping globally on the perception of hardness for various levels of damping. In both experiments, two types of tasks were used: 1) an in-contact task, in which participants started their movement at the object's surface and indented it, and 2) a contact-transition task, in which they first made a free-air movement, before making contact with nonzero impact velocity and indenting the object. In Experiment 3, the difference between the effect of injecting damping globally (i.e., throughout the workspace) and locally (i.e., inside the object only) on the perception of hardness was investigated. In tele-operation systems, damping is usually

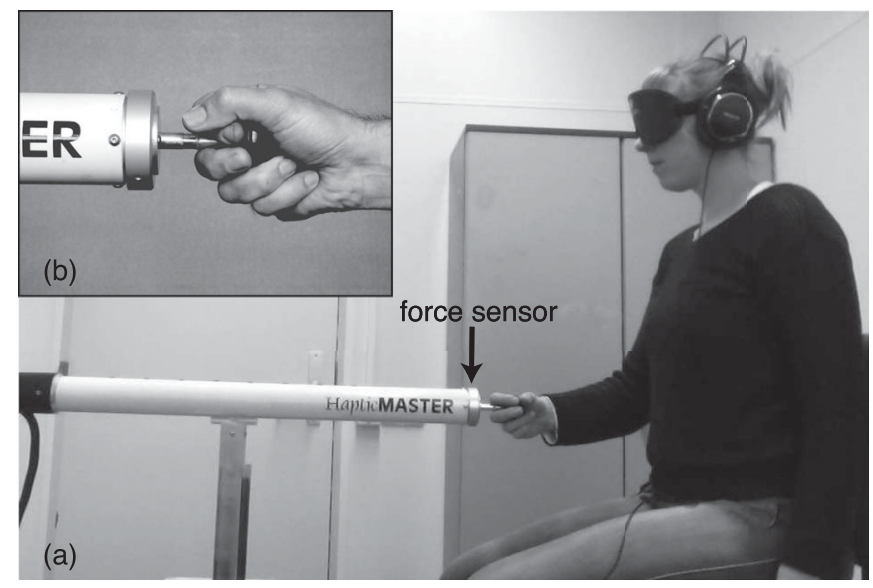

Fig. 1. Overview of the setup. (a) Blindfolded participant holding the handle of the HapticMaster. The location of the force sensor, which is positioned at the base of the handle, is indicated with an arrow. (b) Close-up of a typical example of a participant's hand position.

implemented both inside objects and in free-space, so Experiments 1 and 2 were used to explore the stiffnessdamping space that is relevant for these applications. Experiment 3 enabled us to compare the results from the first two experiments to those from literature on local damping. In the next section, the general methods are explained first, followed by experiment-specific paragraphs.

\section{Material ANd Methods}

\subsection{Participants}

In all experiments, 12 naive participants took part. None participated in more than 1 experiment. In Experiment 1, three males and nine females took part. They were $21 \pm 3$ (mean \pm standard deviation) years old, nine were right-handed and three were left-handed (assessed using the Coren-test for handedness [15]). In Experiment 2, 2 males and 10 females took part. They were $23 \pm 3$ years old, 11 were right-handed and one was left-handed. In Experiment 3, four males and eight females took part. They were $28 \pm 4$ years old, 11 were right-handed and 1 was left-handed. None of the participants had a history of neurological disorders. Prior to the experiment, they received written instructions and signed an informed-consent form. For Experiments 1 and 2, they received a small payment for their participation. All the experiments were approved by the Ethics Committee of the Faculty of Human Movement Sciences (ECB).

\subsection{Protocol}

\subsubsection{General}

During all experiments, participants were sitting, while wearing a blindfold and headphones. The setup (see Fig. 1) was an admittance-controlled haptic device, the HapticMaster (Moog Inc.). This device is capable of rendering very high stiffnesses (maximum $20 \mathrm{kN} / \mathrm{m}$ ), large forces (maximum $250 \mathrm{~N}$ ) and sensing position and forces very precisely (position resolution better than $12 \times 10^{-6} \mathrm{~m}$, force sensitivity $0.01 \mathrm{~N}$ ) [16]. By compensating for internal friction in the control loop, which is updated at $2,500 \mathrm{~Hz}$, the device works frictionlessly. The handle is a ball-shaped object, which is rigidly connected to the device through a metal bar. It had a simulated inertial mass of $3 \mathrm{~kg}$ to avoid instability, while its 
Reference

Test
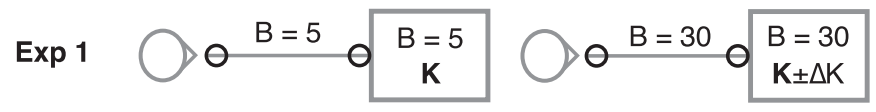

Exp 2
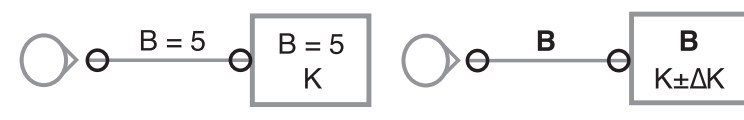

Exp 3
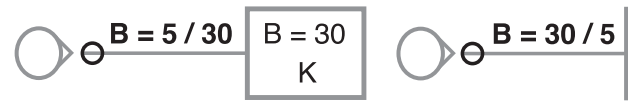

$\mathrm{B}=30$
$\mathrm{~K} \pm \Delta \mathrm{K}$

Fig. 2. Top view of the participant, the trajectory and the virtual object (all in gray) in the three experiments, for the reference and test stimulus. Participants always made movements away from their body, which is from left to right in the figure. In the in-contact task, the start position (indicated with the rightmost black circle for both stimuli in Experiments 1 and 2) was at the object's surface. For the contact-transition task, the participants started their movement $8 \mathrm{~cm}$ closer to their body (the leftmost black circle) and first made a free-air movement (indicated with the gray line) before encountering the object. The numbers show the stiffness $(\mathrm{K})$ and damping (B) values of the reference and the test stimuli in all experiments. Bold numbers indicate the property that was varied across conditions: in Experiment 1, various levels of stiffness were tested for two tasks, in Experiment 2, various levels of damping, injected both inside objects and in free-space (i.e., globally), were tested for two tasks, while in Experiment 3 , a single task was used to compare injecting damping mainly inside objects ('locally') to injecting damping both inside objects and in free-space ('globally'). In all experiments, the stiffness of the test stimulus was varied to find the level at which test and reference stimulus were perceptually equal in hardness.

gravity was compensated for. Headphones worn by the participants produced white noise to mask the sound of the haptic device. Damping was never applied directionally, so irrespective of the movement direction, a high damping force was present for all movements in strongly damped environments.

On each trial, participants were consecutively presented with two virtual objects, each composed of a linear spring. The participants were asked to grab the handle when the white noise started and then move the handle away from them along their sagittal axis, which was the axis to which the movement of the device was restricted. During their movement, they encountered the first object. They were free to move the handle into the virtual object as far as they liked, but they were only allowed to move forward. Once the participants felt they had reached the end of their forward movement, they had to release the handle and the white noise stopped. After this, the handle moved back to the start position, the white noise started again and the participant made the second movement to observe the second object. Once (s)he completed this movement, (s)he had to indicate which object felt harder. Subsequently, a new trial started. Both the start and the object were always located at the same positions. Fig. 2 shows a top-view of the participant, the movement trajectory and the object.

To obtain a fast and precise measure of the difference between heavily damped and lightly damped stimuli, a oneup-one-down staircase procedure was used in which the stiffness of the test stimulus was adapted. For an example of one of the staircases, see Fig. 3a. In a staircase procedure, the properties of the reference stimulus are kept constant, while the properties of the test stimulus are adjusted according to the answers of the participant. In our procedure, we used a reference stimulus with a constant level of stiffness and damping. The test stimulus had another constant damping level, while its test stiffness was adjusted during the experiment. In Experiments 1 and 2, we used 2 interleaved staircases in each condition, which we called a 'staircase pair'. In Experiment 3, we used two interleaved staircase pairs, so two conditions were measured simultaneously. In all experiments, each staircase pair consisted of one staircase with an initial stiffness that was 30 percent higher than that of the reference stimulus and one staircase with a initial stiffness that was 30 percent lower than the reference stiffness. In each trial, one reference and one test stimulus were presented. If the participant answered that the test (reference) stimulus was the harder stimulus, the test stiffness was decreased (increased) on the next trial to find the test stiffness at which the perceptual hardness of the test stimulus was equal to that of the reference stimulus. The step size with which the stiffness was changed was 6 percent of the reference stiffness for all experiments.

When both of the staircases of the staircase pair had reversed at least 5 times, both staircases were terminated. When the maximum of 50 trials was reached, both staircases were also terminated. In Experiments 2 and 3, we used a minimal number of 30 trials per staircase pair. In Experiment 1 , this minimum was not set yet, but usually at least 30 trials were performed in Experiment 1 as well. The order of the conditions was counterbalanced between participants, while the order of test and reference stimulus within each of the staircases was pseudo-randomly assigned, which ensured that both orders were tested equally often. Throughout the experiment, force and position data were recorded with a frequency of $1,024 \mathrm{~Hz}$ using the datalogger function of the HapticMaster. Experiments 1 and 2 took about one hour per participant each, while Experiment 3 took about 30 minutes per participant.

\subsubsection{Experiment 1}

In Experiment 1, the aim was to investigate the effect of injecting damping globally on perceived hardness, for various levels of stiffness. Two types of tasks were used: a contact-transition task and an in-contact task. In the contacttransition task, participants first made a free-air movement of $8 \mathrm{~cm}$ towards the virtual object, before making contact with it. So, in this task, the movement consisted of two parts (while it was made as a single movement by the participant, such that the impact occurred with non-zero velocity): a free-air part and an in-contact part. It was stressed to the participants, both verbally and in the written instruction, that they should not base their decision about the hardness of the virtual object on the free-air part, but rather focus on the impact and the part where they were in contact with the object. In the in-contact task, the free-air part of the movement was eliminated by placing the start position at the surface of the virtual object, so effectively only the in-contact part of the task remained. The rest of the instructions were the same for the two tasks. For a visualization of the task and the conditions, see Fig. 2.

For each task, three reference stiffnesses were used, which were 500, 1,000 and 2,000 N/m for the in-contact task and $1,000,2,000$ and $4,000 \mathrm{~N} / \mathrm{m}$ for the contact-transition task, resulting in 6 conditions ( 3 stiffness levels $\times 2$ tasks). The 

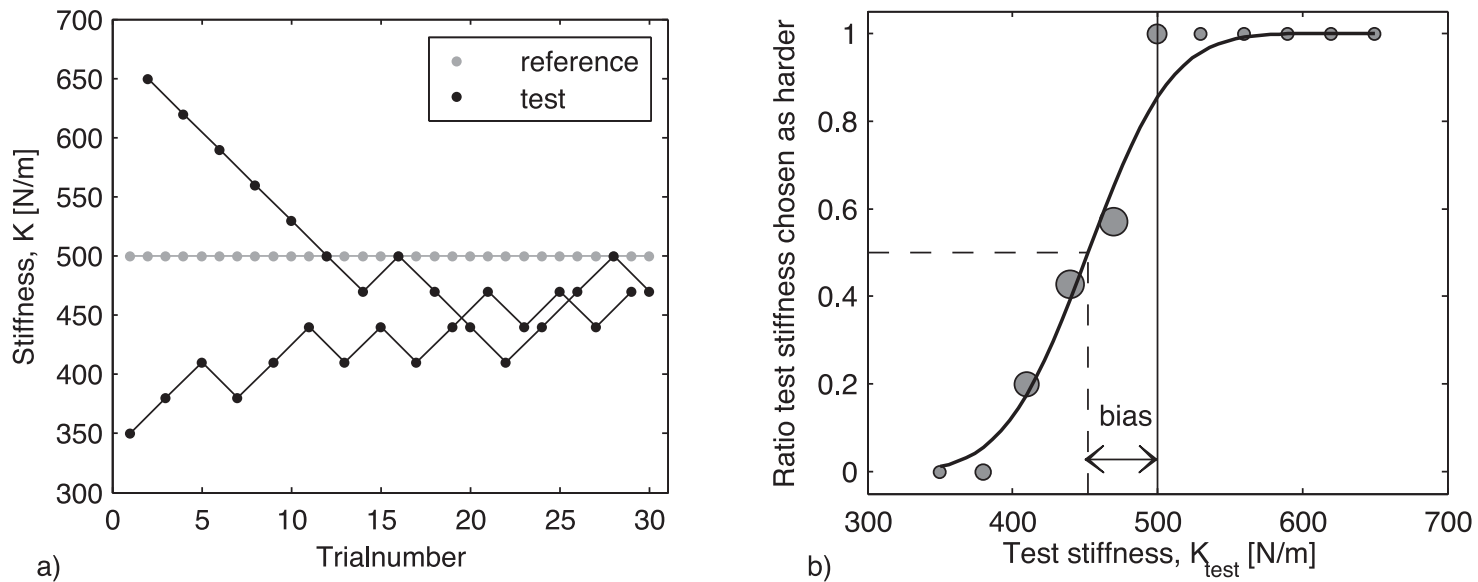

Fig. 3. Typical example of a staircase pair and the psychometric curve fitted to the perceptual data of one participant, for a reference stiffness of $500 \mathrm{~N} / \mathrm{m}$ in an in-contact task in Experiment 1. a) Staircase pair showing one experimental condition. In each trial, one reference stimulus (gray dot) and one test stimulus (black dot) was presented. If the participant perceived the test (reference) stimulus as the harder stimulus, the stiffness of the next test stimulus was decreased (increased) to find the test stiffness at which both stimuli were perceived as equally hard. b) Psychometric function fitted to the perceptual data shown in panel a. Gray dots show actual data points, while the black curve is the psychometric function fitted to the data. The size of the dots shows the number of times this point was measured and therefore its weight in the fit procedure. The vertical black line shows the reference stiffness. The PSE is the value on the horizontal axis corresponding to a ratio of 0.5 , which is indicated with the dashed lines. The bias is the difference between the PSE and the reference stiffness, which is indicated with an arrow.

stiffness ranges differed between the tasks, because we aimed to cover a broad range of hardnesses for both tasks. For the incontact task, it was very difficult to indent an object with a stiffness above 2,000 N/m. For the contact-transition task, it was very difficult to properly feel the transition from free-air movement to object indentation for a stiffness below 1,000 N/ $\mathrm{m}$. Therefore, these different stiffness ranges were selected. On the trials where the reference stiffness was presented, the damping was always $5 \mathrm{Ns} / \mathrm{m}$ (so both inside the object and in free-space, i.e., 'global'). On trials where the test stiffness was presented, the damping was always $30 \mathrm{Ns} / \mathrm{m}$ (again, both inside the object and in free-space, i.e., 'global'). We chose these values after preliminary testing, by selecting a damping value for the test stimulus which provided a considerable amount of damping, while still allowing the user to move comfortably. We did not design the reference stimulus to have no damping, because the device then sometimes became unstable upon object contact for high stiffnesses and velocities. We did include one condition in Experiment 2 in which the test stimulus was undamped, but this condition was not critical for the results.

\subsubsection{Experiment 2}

In Experiment 2, the aim was to investigate the effect of injecting damping globally on perceived hardness, for various levels of damping. Again, a contact-transition task and an incontact task were used. For the in-contact task, the reference stiffness was always $1,000 \mathrm{~N} / \mathrm{m}$. For the contact-transition task, the reference stiffness was always $2,000 \mathrm{~N} / \mathrm{m}$. In each condition, the global damping for the reference stimulus was always $5 \mathrm{Ns} / \mathrm{m}$, while the global damping for the test stimulus differed between blocks: $0,10,15,20$, and $25 \mathrm{Ns} / \mathrm{m}$ were used. This resulted in ten conditions (5 damping levels $\times 2$ tasks). Fig. 2 shows a visual description of these conditions.

\subsubsection{Experiment 3}

In Experiment 3, the aim was to investigate the difference between the effect of injecting damping globally (i.e., throughout the workspace) and locally (i.e., inside the object only) on the perception of hardness. Only a contacttransition task was used, for which the reference stiffness was always $2,000 \mathrm{~N} / \mathrm{m}$. Two interleaved staircase pairs (four staircases) were used to measure two conditions simultaneously. In both staircase pairs, one of the staircases consisted of stimuli that were mainly damped locally, while the other consisted of stimuli that were damped globally. In both types of stimuli, the damping inside the object was always $30 \mathrm{Ns} / \mathrm{m}$. For the latter stimuli, the situation was the same as for the test stimuli in Experiment 1: the damping in free-space was also $30 \mathrm{Ns} / \mathrm{m}$. For the former stimuli, the damping in free-space was $5 \mathrm{Ns} / \mathrm{m}$. In one condition, the reference stimulus was mainly damped locally, while the test stimulus was damped globally. In the other condition, the reference stimulus was damped globally, while the test stimulus was mainly damped locally. So, the only difference between the conditions was which stimulus was the reference and the test. In Fig. 2 these conditions are illustrated visually. The experiment was measured in one block. The participants were asked if they wanted a break when the experiment was about halfway, but none of the participants felt they needed that.

\subsection{Data Analysis}

\subsubsection{Perceptual Data}

Perceptual data were analyzed by determining the bias per condition for each participant. The bias is the difference between the Point of Subjective Equality (PSE) and the reference stiffness. This bias was obtained by first determining all the combinations of reference and test stimuli that were presented for that staircase pair. For each combination, the number of times that the participant responded that the test stimulus was the harder stimulus was counted and divided by the total number of trials in which the combination was presented, resulting in a response ratio. An example of such a data set is shown in Fig. 3b. To calculate the bias, a psychometric function was fitted to the data. For the condition 

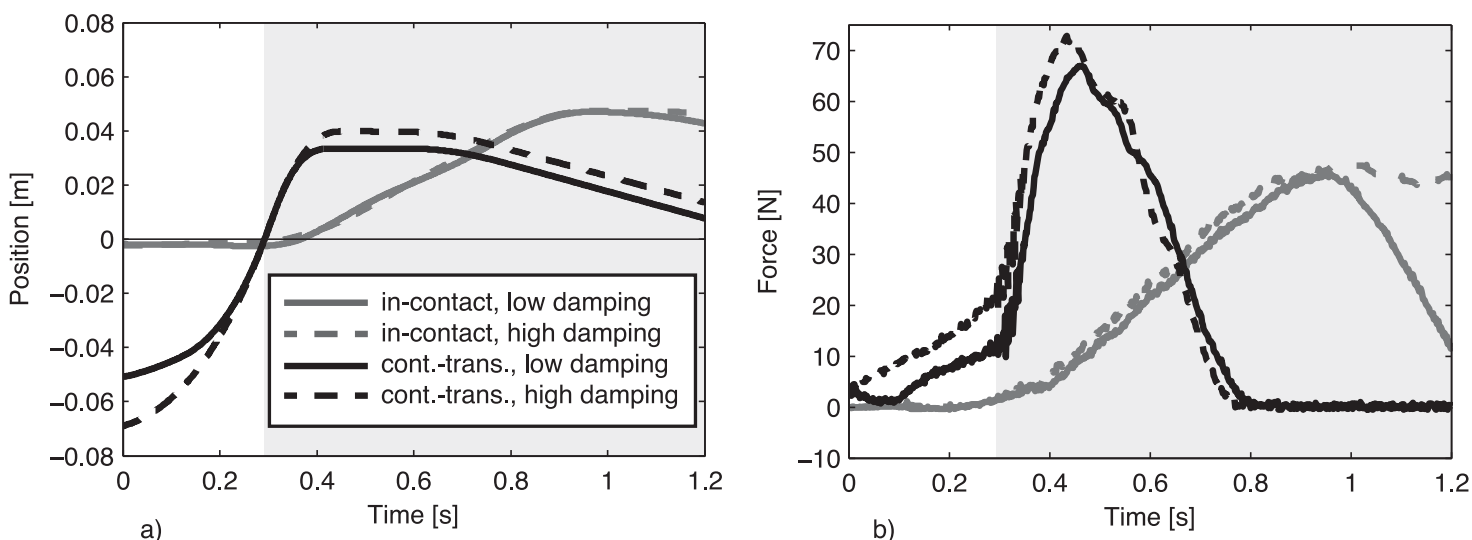

Fig. 4. Typical examples of position data (a) and force data (b) of one in-contact trial (gray, stiffness for both movements $1,000 \mathrm{~N} / \mathrm{m}$ ) and one contacttransition trial (black, stiffness for both movements $2,000 \mathrm{~N} / \mathrm{m}$ ). The solid lines show movements in the low-damped environment $(5 \mathrm{Ns} / \mathrm{m})$, while the dashed lines show movements in the high-damped environment (30 Ns/m). The object surface was placed at position 0 , so the gray background shows the part of the movement in contact with the object. Note that for the in-contact task, the high-damped movement yields larger forces than the low-damped movement, while the displacement profile is similar. For the contact-transition task, initial forces are higher for the high-damped movement, but the force increase upon object contact is almost similar, while the indentation is larger for the high-damped movement.

in Experiment 2 in which the test stimulus had no damping and was therefore more lightly damped than the reference stimulus, the sign of the bias was reversed. This was done to make sure that the bias represented the difference between the more lightly and more heavily damped stimulus in a consistent manner. The following equation was used to describe the relation between test stiffness $K_{\text {test }}$ and measured response ratio, with fitting parameters bias $(\mu)$ and JND $(\sigma)$ :

$$
f\left(K_{\text {test }}\right)=\frac{1}{2}+\frac{1}{2} \operatorname{erf}\left(\frac{K_{\text {test }}-K_{\text {ref }}-\mu}{\sqrt{2} \sigma}\right) .
$$

An extra analysis of the data showed that using the last 6 reversals as a measure of the bias resulted in biases similar to the ones obtained using our procedure. To assess the goodness of fit to the measured data, the $R^{2}$ was calculated for each fit. When the $R^{2}$ was smaller than 0.25 , the fit was deemed too poor and the bias was not used in further analyses. This was the case for 5 of the 72 calculated biases ( 7 percent) in Experiment 1, for 2 of the 110 calculated biases (2 percent) in Experiment 2, and for 2 of the 24 calculated biases (8 percent) in Experiment 3. In Experiment 2, one participant indicated at the debriefing that she had guessed the purpose of the experiment and therefore had manipulated her answers to make sure that she was 'not being fooled'. Therefore, her data were discarded from the analysis, resulting in a total of 11 participants in Experiment 2. The remaining biases were averaged over participants per task and reference stiffness. To assess if the biases differed significantly from 0 , a Student's $t$-test was performed on the biases of each condition. For Experiment 1, the effect of reference stiffness level on the global damping-induced biases was evaluated for both tasks separately, using a repeated measures ANOVA with reference stiffness level as withinsubject factor. For Experiment 2, the effect of damping level on the global damping-induced biases was evaluated for both tasks separately, using a repeated measures ANOVA with damping level as within-subject factor. When the sphericity-criterion was not met, Greenhouse-Geisser correction was used. Note that in case of a goodness-of-fit being too poor and thus discarding the data from the analysis, all biases for that participant are also automatically discarded from the ANOVA analysis of that task. For Experiment 3, the difference between the biases in both conditions was assessed using a paired $t$-test.

\subsubsection{Position and Force Data}

Position and force data were recorded to investigate if there were parameters that correlated to the perceptual experience of the participants, for instance, if participants always perceived a trial in which the force was higher as being the harder stimulus. Fig. 4 shows an example of these data for one trial. If biases in the perceptual data are present, the position and force parameters could indicate which parameters participants used to base their percept on. Furthermore, a difference between tasks in the correlation of parameters and perceptual decision could indicate that participants switched strategies between tasks. For each trial, the following characteristics were calculated:

Free-air phase:

- Movement time until contact [s]

- Mean movement velocity $\left[\frac{\mathrm{m}}{\mathrm{s}}\right]$

Impact phase:

- Impact velocity $\left[\frac{\mathrm{m}}{\mathrm{s}}\right]$

- Impact force [N]

- Extended rate-hardness $\left[\frac{\mathrm{N} / \mathrm{s}}{\mathrm{m} / \mathrm{s}}\right]$

In-contact phase:

- Movement time during indentation [s]

- Maximum object indentation [m]

- Mean movement velocity $\left[\frac{\mathrm{m}}{\mathrm{s}}\right]$

- Peak movement velocity $\left[\frac{\mathrm{m}}{\mathrm{s}}\right]$

- Mean deceleration during indentation $\left[\frac{\mathrm{m}}{\mathrm{s}^{2}}\right]$

- Peak deceleration during indentation $\left[\frac{\mathrm{m}}{\mathrm{s}^{2}}\right]$

- Mean force [N]

- Peak force [N]

- Force difference between impact and maximum object indentation $[\mathrm{N}]$

- Adjusted rate-hardness $\left[\frac{\mathrm{N} / \mathrm{s}}{\mathrm{m} / \mathrm{s}}\right]$ 


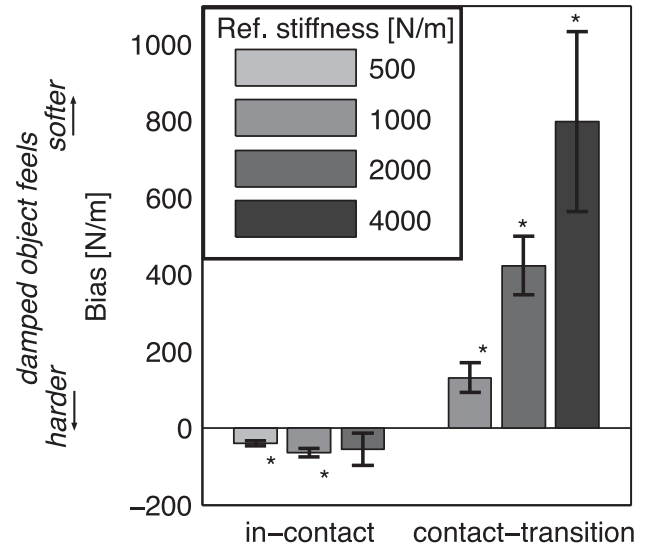

(a)

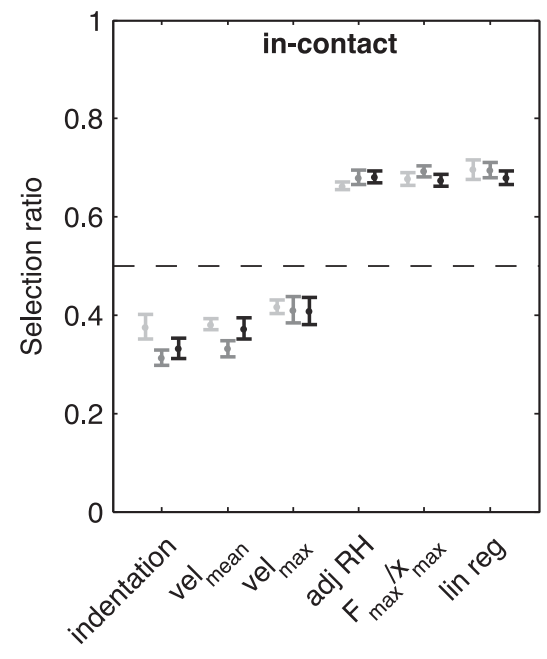

(b)

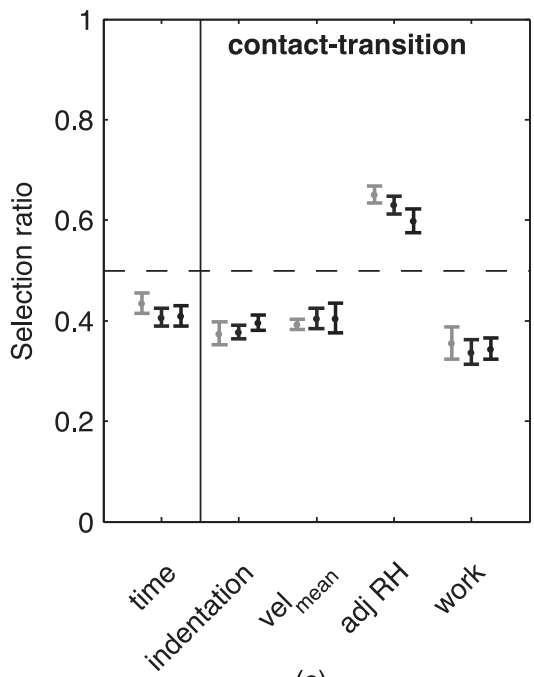

(c)

Fig. 5. Results of Experiment 1. (a) Biases for both tasks, averaged over participants. The error bars show \pm 1 standard error, while the gray values correspond to the various reference stiffnesses. A positive (negative) bias means that the heavily damped object is perceived as softer (harder) than the lightly damped object. Note that heavily damped objects were perceived as harder in the in-contact task, while they were perceived as softer in the contact-transition task. All biases differed significantly from 0 , except for the bias of the in-contact task with a reference stiffness of $2,000 \mathrm{~N} / \mathrm{m}$. ${ }^{*} p<0.05$ when comparing bias to 0 . (b) Parameters for in-contact task from movement and force data that correlated significantly with choices in the perceptual task, which means that they differed significantly from the chance level of 0.5 for all reference stiffnesses. When the ratio is larger (smaller) than 0.5, participants were inclined to connect an increase in this parameter to the object feeling harder (softer). Gray values correspond to the different reference stiffnesses, while error bars show \pm 1 standard error. (c) Significant parameters for contact-transition task. The vertical black line separates the parameters in the free-air phase from parameters in the in-contact phase. No parameters were significantly different from 0.5 for all stiffness levels in the impact phase.

- Peak force/peak indentation $[\mathrm{N} / \mathrm{m}]$

- Mean force difference/indentation $[\mathrm{N} / \mathrm{m}]$

- Slope of the linear regression of force as a function of position $[\mathrm{N} / \mathrm{m}]$

- Work [J]

Extended rate-hardness is defined as the maximum rate of change of force divided by the movement velocity at impact. This parameter was meaningless in our impact-free incontact task, so we introduced a new parameter, which we call 'adjusted rate-hardness'. This parameter was calculated by fitting straight lines to the force and position data as a function of time, between impact and maximum object indentation. The ratio of the two fitted slopes was called 'adjusted rate-hardness'. Note that the unit of adjusted and extended rate hardness, $\left[\frac{\mathrm{N} / \mathrm{s}}{\mathrm{m} / \mathrm{s}}\right]$, could be simplified to $\left[\frac{\mathrm{N}}{\mathrm{m}}\right]$. However, this was not done for extended rate-hardness in the original definition [12]. For consistency and to avoid confusion with a pure stiffness, the unit $\left[\frac{\mathrm{N} / \mathrm{s}}{\mathrm{m} / \mathrm{s}}\right]$ was used for both extended and adjusted rate-hardness. When no damping is present, rate-hardness and stiffness are the same, but for the movements in which there is damping, a substantial additional force is present, such that rate-hardness and stiffness are no longer equivalent. The parameters for the incontact phase were calculated for both tasks, while the parameters for the free-air phase and the impact phase were only used for the contact-transition task, as they had no meaning in the in-contact task. When a derivative was needed to calculate the parameter, the difference data were filtered using a 4th-order Butterworth low-pass filter with a $500 \mathrm{~Hz}$ cut-off frequency. The linear regression metric was based on Nisky et al. [17], while the work metric was calculated as described in Di Luca et al. [18].

The calculated parameters were grouped based on the perceptual data: when participants answered that the first stimulus was the harder one, the parameter from the second trial was subtracted from that of the first and vice versa. When the difference score was positive, a ' 1 ' was scored, while for negative scores a ' 0 ' was noted. For each task, reference stiffness and participant, the scores of all trials were summed and divided by the total number of trials, resulting in a score between 0 and 1 , which we called 'selection ratio'. The chance value would be 0.5 , while a significant deviation from 0.5 would indicate a correlation between hardness perception and the measured parameter. For instance, a value above 0.5 would indicate that participants chose the stimulus that scored higher on this characteristic more often as the harder stimulus. Student's $t$-tests were used to assess if the parameters indeed deviated from 0.5. Only parameters that significantly differed from 0.5 for all conditions within an experiment are shown in the Results section, since they are correlated to the perceptual experience most strongly and consistently. The position and force data from participants and conditions in which the perceptual bias could not be determined reliably enough, as described in the subsection 'Perceptual data', were omitted from the analysis.

\section{Results}

\subsection{Experiment 1}

\subsubsection{Perceptual Data}

All the biases measured in Experiment 1 are shown in Fig. 5a. These biases were based on $30 \pm 0.75$ trials (mean \pm standard error) per staircase pair. The in-contact task yielded negative biases, as shown in Table 1, while the contact-transition task yielded positive biases, as shown in Table 2 . Almost all the biases differed significantly from 0 
TABLE 1

Biases and Statistics for the In-Contact Tasks in Experiments 1 and 2

\begin{tabular}{cccccc}
\hline Exp. & K [N/m] & B [Ns/m] & bias \pm s.e. [N/m] & $t$-value & $p$-value \\
\hline \multirow{3}{*}{1} & 500 & & $-39 \pm 6$ & $t_{11}=-6.4$ & $\leq 0.001^{*}$ \\
& 1000 & 30 & $-64 \pm 12$ & $t_{11}=-5.4$ & $\leq 0.001^{*}$ \\
& 2000 & & $-56 \pm 42$ & $t_{11}=-1.3$ & 0.21 \\
\hline \multirow{4}{*}{2} & & 0 & $-20 \pm 11$ & $t_{10}=-1.9$ & 0.092 \\
& & 10 & $-17 \pm 12$ & $t_{10}=-1.5$ & 0.17 \\
& & 15 & $-6.3 \pm 12$ & $t_{10}=-0.51$ & 0.62 \\
& & 20 & $-19 \pm 9$ & $t_{10}=-2.0$ & 0.073 \\
& & 25 & $-21 \pm 12$ & $t_{10}=-1.7$ & 0.12 \\
\hline
\end{tabular}

${ }^{*} p<0.05$ for a one-sample $t$-test with a test value of 0 .

(Tables 1 and 2 show the exact values). The repeated measures ANOVAs showed a significant effect of reference stiffness on bias size in the contact-transition task $\left(F_{2,12}=6.8\right.$, $\left.p=0.011, \eta_{p}^{2}=0.53\right)$, while there was no effect in the in-contact task $\left(F_{2,22}=0.23, p=0.80, \eta_{p}^{2}=0.021\right)$. The different sign of the bias means that in the in-contact task, perceived hardness was increased when damping was injected globally, while for the contact-transition task, perceived hardness was decreased in the same condition. Since almost all the biases differed significantly from 0 , this implies that the biases also differ significantly between the tasks. The results of Experiment 1 have been published previously in conference proceedings [14].

\subsubsection{Position and Force Data}

The position and force data of Experiment 1 (see Fig. 5b-c) showed 6 parameters that significantly differed from chance for all of the reference stiffnesses in the in-contact task, which were: maximum indentation, mean velocity, maximum velocity, adjusted rate-hardness, maximum
TABLE 2

Biases and Statistics for the Contact-Transition Tasks in Experiments 1, 2, and 3

\begin{tabular}{|c|c|c|c|c|c|}
\hline Exp. & $\mathrm{K}[\mathrm{N} / \mathrm{m}]$ & $B[\mathrm{Ns} / \mathrm{m}]$ & bias \pm s.e. $[\mathrm{N} / \mathrm{m}]$ & $t$-value & $p$-value \\
\hline \multirow{3}{*}{1} & 1000 & & $131 \pm 39$ & $t_{11}=3.4$ & $0.006^{*}$ \\
\hline & 2000 & 30 & $423 \pm 76$ & $t_{10}=5.6$ & $\leq 0.001^{*}$ \\
\hline & 4000 & & $798 \pm 234$ & $t_{7}=3.4$ & $0.011^{*}$ \\
\hline \multirow{5}{*}{2} & & 0 & $20 \pm 41$ & $t_{10}=0.48$ & 0.64 \\
\hline & & 10 & $100 \pm 27$ & $t_{10}=3.7$ & $0.004^{*}$ \\
\hline & 2000 & 15 & $146 \pm 72$ & $t_{10}=2.0$ & 0.071 \\
\hline & & 20 & $149 \pm 70$ & $t_{9}=2.1$ & 0.062 \\
\hline & & 25 & $223 \pm 58$ & $t_{9}=3.9$ & $0.004^{*}$ \\
\hline \multirow[t]{2}{*}{3} & 2000 & test global & $400 \pm 130$ & $t_{10}=3.1$ & $0.012^{*}$ \\
\hline & & test local & $-170 \pm 80$ & $t_{10}=-2.3$ & $0.046^{*}$ \\
\hline
\end{tabular}

p $p<0.05$ for a one-sample $t$-test with a test value of 0 .

force divided by maximum indentation, and the linear regression of force on position (all $p \leq 0.0068$ ). For the contact-transition task, the following five parameters differed significantly from chance for all the reference stiffnesses: movement time during the free-air phase, maximum object indentation, mean velocity, adjusted rate-hardness, and work (all $p \leq 0.034$ ). The latter 3 parameters concerned the in-contact phase of the movement.

\subsection{Experiment 2}

\subsubsection{Perceptual Data}

All the biases measured in Experiment 2 are shown in Fig. 6a. These biases were based on $32 \pm 0.31$ trials (mean \pm standard error) per staircase pair. The in-contact task yielded negative biases, as shown in Table 1, while the contact-transition task yielded positive biases, as shown in Table 2. For the in-contact task, none of the biases differed

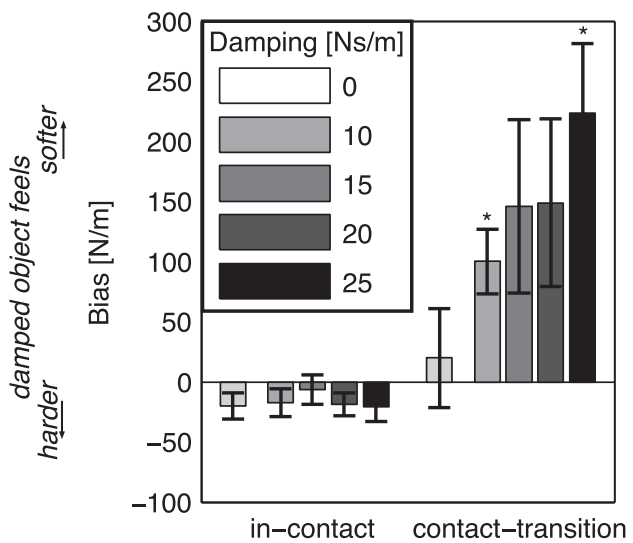

(a)

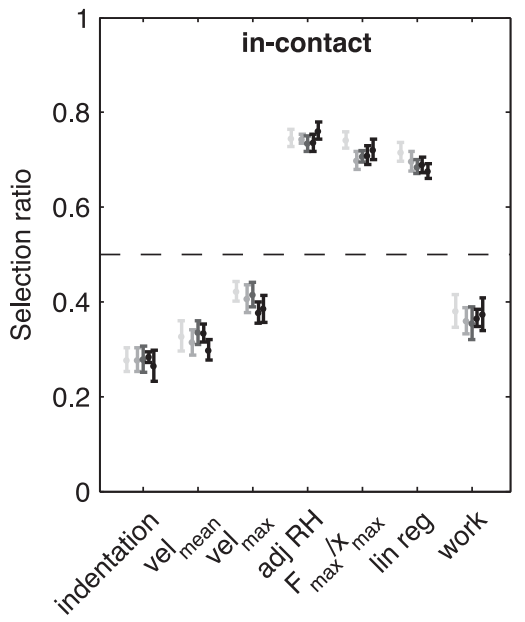

(b)

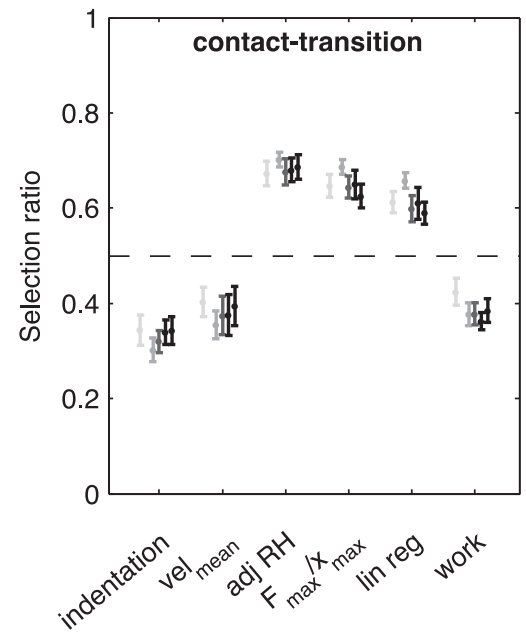

(c)

Fig. 6. Results of Experiment 2. (a) Biases for both tasks, averaged over participants. The error bars show \pm 1 standard error, while the gray values correspond to the various levels of damping of the test stimulus. For the in-contact task, the reference stiffness was always $1,000 \mathrm{~N} / \mathrm{m}$, while it was always $2,000 \mathrm{~N} / \mathrm{m}$ for the contact-transition task. Note that heavily damped objects were perceived as harder in the in-contact task, while they were perceived as softer in the contact-transition task. The biases for 10 and $25 \mathrm{Ns} / \mathrm{m}$ differed significantly from 0 in the contact-transition task. ${ }^{*} p<0.05$ when comparing biases to 0 . (b) Parameters for in-contact task from movement and force data that correlate significantly with choices in the perceptual task. Gray values correspond to the different damping levels, while error bars show \pm 1 standard error. (c) Significant parameters for contact-transition task. No parameters were significantly different from 0.5 for all damping levels in the free-air phase or the impact phase. See Fig. 5 for a more elaborate caption. 
significantly from 0 , while some biases did differ from 0 in the contact-transition task (Tables 1 and 2 show the exact values). The repeated measures ANOVAs showed a significant effect of damping level on bias size in the contacttransition task $\left(F_{4,32}=3.9, p=0.011, \eta_{p}^{2}=0.33\right)$, while there was no effect in the in-contact task $\left(F_{4,40}=2.4, p=0.062\right.$, $\left.\eta_{p}^{2}=0.20\right)$. Similar to Experiment 1, biases for the in-contact task were negative, while biases for the contact-transition task were positive, implying a difference between the tasks when injecting damping globally.

\subsubsection{Position and Force Data}

The position and force data of Experiment 2 (see Fig. 6b-c) showed 7 parameters that differed significantly from chance for all conditions in the in-contact task, which were: maximum indentation, mean velocity, maximum velocity and adjusted rate-hardness, maximum force divided by maximum indentation, the linear regression of force on position, and work (all $p \leq 0.0093$ ). For the contact-transition task, the following 6 parameters differed significantly from chance for all conditions: maximum object indentation, mean velocity, adjusted rate-hardness, maximum force divided by maximum indentation, the linear regression of force on position, and work (all $p \leq 0.037$ ). All the parameters concerned the in-contact phase of the movement.

\subsection{Equal Hardness Lines}

The aim of Experiment 1 was to investigate the influence of injecting damping globally (i.e., equally both inside the object and in free-space) on perceived hardness for different levels of stiffness, while Experiment 2 had the same aim for different levels of damping. In both experiments, the two different tasks were used. To combine all the information obtained in the two experiments, Fig. 7 was created. In the left panel, the information for the in-contact task in both experiments is summarized, while in the right panel, the data from the contact-transition task are shown. Along the vertical direction, the influence of reference stiffness can be seen, while along the horizontal direction, the influence of damping is shown. Note that the dotted lines only connect equal hardness pairs, they are not a suggestions of the shape of the connecting lines. The solid lines do indicate the effect of damping on perceived hardness, as the data of Experiments 1 and 2 together do provide enough data to discern a relation between damping level and perceived hardness for the reference stiffness of 1,000 $\mathrm{N} / \mathrm{m}$ for the in-contact task and for the reference stiffness of $2,000 \mathrm{~N} / \mathrm{m}$ for the contact-transition task. For the incontact task, the shape of the data most closely resembled a linear relation, so a linear function was fitted to the data to describe an equal hardness line, resulting in an $R^{2}$ of 0.78 . For the contact-transition task, the shape most closely resembled a power function, which was fitted to that part of the data to describe the equal hardness line, resulting in an $R^{2}$ of 0.91 . A linear fit to the latter data resulted in an $R^{2}$ of 0.89 , so fitting a power function was indeed slightly more appropriate for the contact-transition task. So, the panels of Fig. 7 provide an image of pairs of damping and stiffness values which resulted in an equal sensation of hardness in the two different tasks in these experiments.

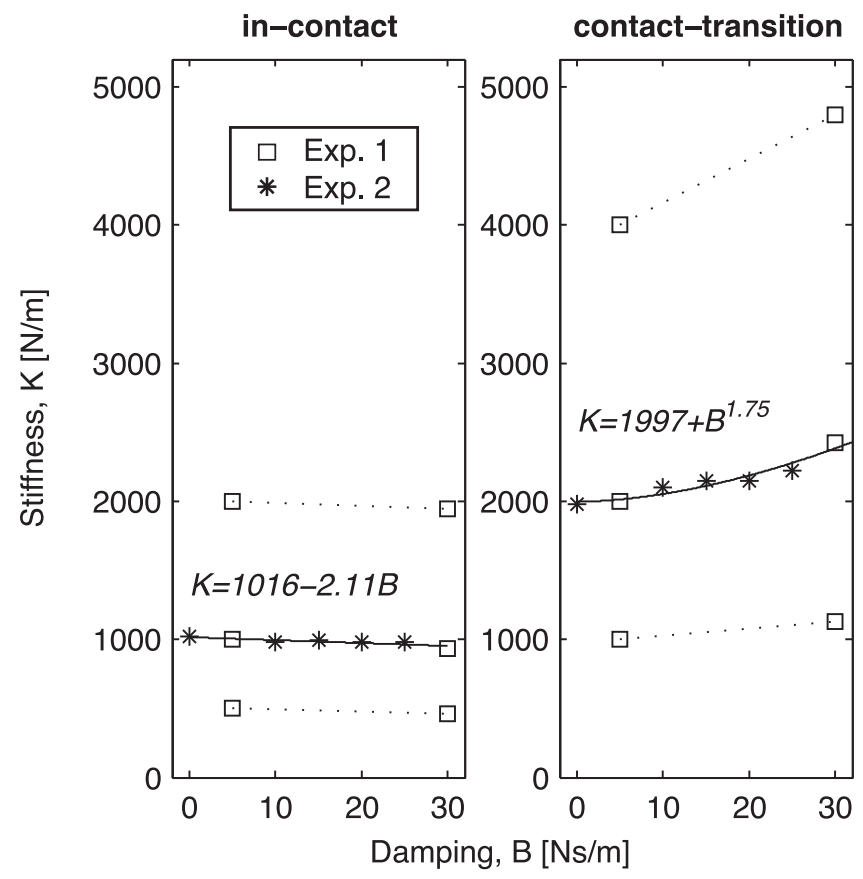

Fig. 7. Equal hardness lines, for the in-contact task (left) and the contacttransition task (right), from Experiments 1 and 2. Note that the dotted lines only connect stiffness-damping pairs with equal perceptual hardness, but they are not intended as an interpolation between the two pairs. The fitted solid lines do show an interpolation, based on a combination of some data of Experiment 1 and all data of Experiment 2. For the in-contact task, the increase in perceived hardness (stronger negative bias), can be described using a linear fit. For the contact-transition task, a power function is more appropriate to describe the decrease in perceived hardness (stronger positive bias).

\subsection{Experiment 3}

\subsubsection{Perceptual Data}

Fig. 8a shows an overview of the biases that were measured in the two interleaved conditions in Experiment 3. These biases were based on $32 \pm 0.18$ trials (mean \pm standard error) per staircase pair. For the condition in which the test stimuli were damped globally, while the reference stimuli were damped locally, the measured bias was positive. For the condition in which the test stimuli were damped locally, while the reference stimuli were damped globally, the measured bias was negative. Both biases differed significantly from 0 (see Table 2), which implies that the biases also differ from each other. This shows the consistency of the effect: when the test stimulus was damped globally, it was perceived as softer than the locally damped reference stimulus, while when the test stimulus was damped locally, it was perceived as harder than the reference stimulus that was damped globally. However, a paired $t$-test showed that the magnitude of the biases did differ significantly $\left(t_{10}=2.9, p=0.017\right)$.

\subsubsection{Position and Force Data}

Fig. $8 \mathrm{~b}$ shows that four parameters in the force and position data differed significantly from chance for both conditions in Experiment 3: maximum indentation, adjusted ratehardness, maximum force divided by maximum indentation, and work (all $p \leq 0.038$ ). All significant parameters concerned the in-contact phase of the movement. During the free-air movement and the impact phase, none of the parameters differed significantly from 0 in both conditions. 


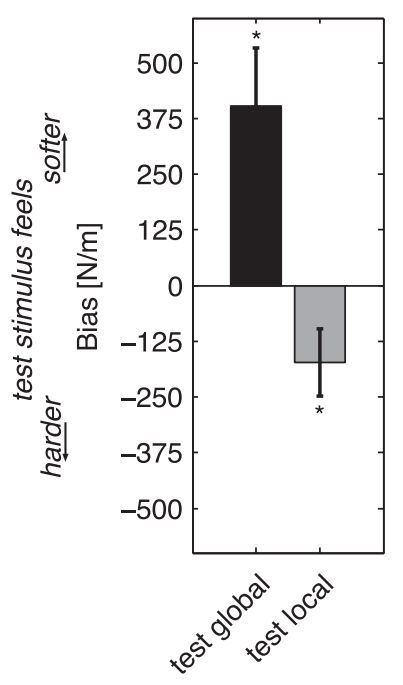

(a)

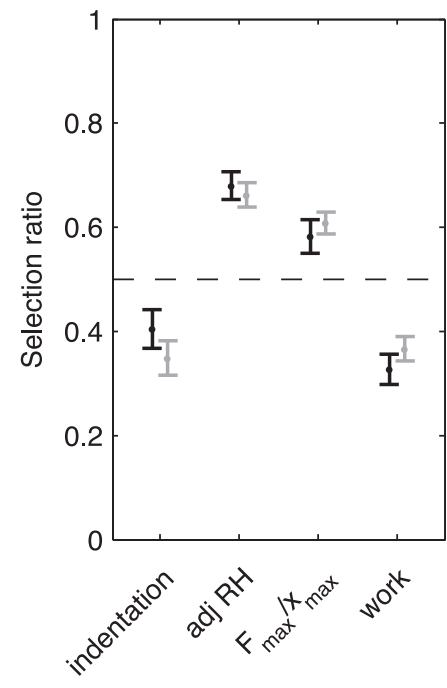

(b)
Fig. 8. Results of Experiment 3. (a) Biases for both conditions, averaged over participants. The error bars show \pm 1 standard error. The labels on the horizontal axis indicate the type of damping of the test stimulus. The biases had a different direction, and differed significantly from 0 . This indicates the consistency of the effect: when the test stimulus is damped globally, it feels softer than the reference stimulus that was damped locally, while when the test stimulus is damped locally, it feels harder than the reference stimulus that is damped globally. ${ }^{*} p<0.05$ when comparing biases to 0 . (b) Parameters from movement and force data that correlated significantly with choices in the perceptual task. Gray values correspond to the different damping conditions, while the error bars represent \pm 1 standard error. See Fig. 5 for a more elaborate caption.

\section{Discussion}

In this study, a large effect of damping on perceived hardness was found. Interestingly, this effect was very different for the two experimental tasks: adding damping increases the perceived hardness for an in-contact task, while it decreases it for a contact-transition task. This effect was found in both Experiment 1 and Experiment 2. Experiment 3 indicated that this effect was much larger when the damping was present both inside the object and in free-space (i.e., globally), compared to when it was present inside the object only (i.e., locally).

Apart from the direction of the effect being taskdependent, the size of the effect is also very different between the tasks: the decrease for the contact-transition task was much larger than the increase for the in-contact task, when using global damping, as shown in Experiment 1. The highest damping level resulted in a relative bias of $\sim 20$ percent for the contact-transition task, which is close to the JND of $\sim 23$ percent for stiffness [19]. This means that this stiffness difference would be (close to) perceivable in an undamped situation. Moreover, the bias increased with increasing reference stiffnesses, indicating that the magnitude of the bias could be a fixed percentage of the reference stiffness, which would result in increasing absolute biases with increasing reference stiffnesses. In Experiment 2, the same difference in effect size between the tasks was found. For this experiment, the decrease for the in-contact task was not significantly different from 0 for any of the damping levels, while it was positive for the contact-transition task. Apparently, a damping level of $30 \mathrm{Ns} / \mathrm{m}$ was needed to produce a significant bias in the in-contact task, while a damping level of $10 \mathrm{Ns} / \mathrm{m}$ was enough to produce a significant bias in a contact-transition task. So, while Lawrence et al. [11] argue that dampers could be used to provide a haptic illusion of hard objects, our results show that this is very task-dependent and could even decrease perceived hardness in a contact-transition situation. Contact-transition situations occur frequently in bilateral tele-operation [20], so for these applications, injecting damping globally should be done carefully.

To understand where the perceptual differences between the tasks come from, the force and position data can provide relevant information, as they can give insight into the parameters on which participants based their perceptual decision. In all experiments and in all conditions, maximum object indentation and adjusted rate-hardness were significantly correlated with the perceptual experience of hardness. Mean velocity was significantly correlated in all conditions of Experiments 1 and 2, but not in Experiment 3 . In most Experiments and tasks, the ratio between maximum force and indentation, the linear regression of force on position and the work parameter were also significantly correlated with hardness perception. The direction of the correlation was always the same, so a smaller maximum indentation, mean velocity, and amount of work were always correlated with an object feeling harder, while a smaller adjusted rate-hardness, maximum force-indentation ratio and linear regression slope were always correlated with an object feeling softer. Interestingly, in almost all conditions, only parameters describing the in-contact part of the movement were significantly correlated to the perceptual decision. We had expected parameters describing the impact phase of the movement to be important in the contact-transition task, such as impact force or extended rate hardness, as it is known that contact transients can be very important for simulating hard surfaces [21]. Apparently, our participants followed the instruction to focus on the in-contact part of the movement. Moreover, in both tasks almost the same parameters came out as significant in the same direction, even though the direction of the perceptual effect was reversed between the two tasks. So, it seems that participants did not use different strategies in the different tasks, but there was an actual difference in the task dynamics. Intuitively, this does make sense. When adding damping in an in-contact task, an extra force is added during indentation. So, if a participant used a similar force profile for the two movements in a trial (for a typical example of what the force profiles look like, see Fig. 4b), the damping would decrease the amount of object indentation, making the damped object feel harder. For a contact-transition task, adding damping would make the transition from free-air motion to object indentation a bit more gradual, which would allow the participant to indent the damped object a bit further when using comparable force profiles for the two movements in a trial, making the globally damped object feel softer. The notion that no force parameters were significantly correlated to the perceptual experience strengthens the hypothesis that participants tried to use the same force profile in both parts of the trial. When using this strategy, they could either have assessed the kinematic response of the system (if they mainly used movement parameters to 
base their decision on) or the relation between the force profile and the kinematic response (if they mainly used parameters relating force and position to base their decision on).

To get a grasp on the interplay between stiffness and damping in hardness perception, we constructed the 'equal hardness lines'. These lines illustrate that for the contacttransition task, increasing the level of global damping or increasing the reference stiffness increased the bias in perceived hardness. For the in-contact task, no significant effect of reference stiffness or damping level on perceptual bias was found. However, when performing a linear fit on the biases from the in-contact task, shown in the left graph of Fig. 7, the slope is negative, which suggests that increasing the level of damping does increase the bias. However, all biases were very small in this task, and only the maximum level of damping created a significant bias. The two panels together can be seen as a start of a guideline, which indicates the effect of injecting damping throughout a system on the object hardness perceived by the user of the system. However, the extreme task-dependency of the effect, shown by the reverse of the bias for the different tasks, already indicates that it is virtually impossible to provide one guideline to fit all situations. Nonetheless, the size of the error bars in, for instance, Fig. 6 also indicates that the size of the bias was quite different among participants, even though the direction of the effect was very consistent. It could be suggested that injecting damping globally in a situation where mainly in-contact situations are present probably would not affect the perceived hardness very strongly. In contact-transition tasks, adding damping globally could require alternative adjustments, such as an increase in feedback gain, in order to provide the same perception of hardness. An alternative solution, which is also the safest one, would be to avoid the injection of global damping in environments that require contact-transitions as much as possible. Finally, it is important to note that in natural object manipulation, local surface deformation is a very important cue for object hardness [22], [23]. So, our results cannot be extended directly to a natural situation. However, in haptic devices, which are often being used in tele-operation systems, there is usually no sensory information about local surface deformation, as the rigid interface only provides global force and movement information.

It is striking that existing literature describes an increase in perceived hardness when adding damping to a system, even though tasks were used that are comparable to our contact-transition task [11], [12]. However, there are important differences between their experiments and ours. Firstly, their experiments involved only very small indentations, because of the nature of the task and the devices that were used. In our task, indentation was substantial: in most trials it was in the order of centimeters. From the perspective of information, it is likely that in our experiment, participants were mostly using information acquired during object indentation, which is probably governed by kinaesthetic information. In a tapping task such as the tapping task in Han et al. [12], the moment of impact might be a more salient cue, which is probably more related to cutaneous information, provided by high-frequency sensitive receptors. In Lawrence et al. [11] the used object stiffnesses were so high compared to the limited force production capabilities of their device, that indentation was probably very hard to perceive. Therefore, cutaneous information was probably also the most important source of information for their experiment. If the decrease of hardness in an in-contact task, as found in the current study, is an effect that mainly relies on kinaesthetic information, this could explain why the previous studies did not report this effect.

Secondly, both Lawrence et al. and Han and Choi used a form of local damping in their experiments. Our Experiment 3 clearly shows a large perceptual difference between injecting damping globally and locally: when injecting damping globally for test stimuli, we found a positive bias, while we found a negative bias for test stimuli that were damped locally. These results are congruent with the positive biases for the in-contact task in Experiments 1 and 2, since the test stimuli were also damped globally there. The inversion of the bias with inversion of the reference stimulus shows the consistency of the effect: irrespective of which stimulus was deemed test stimulus, the stimulus that was damped globally was perceived as softer than the locally damped one. The magnitude of the bias for test stimuli that were damped locally was smaller than that for test stimuli that were damped globally, but the mean stiffness during the experiment was also smaller for the former condition, because the reference stiffness was the same in both conditions. Since a staircase converges onto the bias, the mean stiffness of the stimuli depends on the measured bias. Experiment 1 already showed that the size of the bias depends on the stiffness of the objects, so a smaller mean stiffness should result in a smaller bias. Concluding, it seems that, even though other factors like device inertia, maximum force and amount of indentation could still play a role, the difference between injecting damping globally and locally is at least an important part of the explanation of the difference between previous results and our results. Interestingly, the results of Experiment 3 for the condition in which the test stimulus was damped globally and the reference was damped locally are very comparable to the biases found in Experiment 1. In the latter case, the test stimulus was also strongly damped inside the object and in free-space, but the reference stimulus only had very small damping inside the object and in freespace. So, it appears that injecting a large amount of local damping has a comparable effect to injecting a small amount of global damping. Especially for the application in teleoperation systems, results on injecting damping globally are very relevant, since the injection of damping is often used to increase system stability (see e.g., [4], [5], [6]).

\section{CONCLUSION}

We found that injecting damping globally increases the perceived hardness of an object composed of a spring in an incontact task, while it decreases the perceived hardness in a contact-transition task. The latter effect is larger than the former. Participants seem to have used the same strategy in both tasks: using the same force profile during the two movements of one trial and assessing the reaction of the system to this force. Object indentation, mean velocity, work, forceindentation ratio, force-position slope and adjusted ratehardness were closely related to the perceptual experience of hardness. This knowledge could be used by designers of 
teleoperation systems, as they can assess the effect of design choices on the reflected object hardness by their controllers, given the task and required amount of injected damping. Our 'equal hardness lines' could be used as a preliminary guideline in this process. Concluding, our results clearly show that using a damper to increase the perceived hardness of virtual objects is only effective in tasks without impact, while it is detrimental in contact-transition tasks.

\section{ACKNOWLEDGMENTS}

The authors would like to thank Jos van den Berg (Department of Human Movement Sciences, Vrije Universiteit Amsterdam) and Piet Lammertse (Moog Inc.) for their help with HapticMaster. This research is part of the H-Haptics program and is supported by the Dutch Technology Foundation STW, which is part of the Netherlands Organisation for Scientific Research (NWO) and partly funded by the Ministry of Economic Affairs, Agriculture, and Innovation.

\section{References}

[1] D. Lawrence, "Stability and transparency in bilateral teleoperation," IEEE Trans. Robot. Autom., vol. 9, no. 5, pp. 624-637, Oct. 1993.

[2] T. Sheridan, "Space teleoperation through time delay: Review and prognosis," IEEE Trans. Robot. Autom., vol. 9, no. 5, pp. 592-606, Oct. 1993.

[3] E. Nuño, L. Basañez, R. Ortega, and M. W. Spong, "Position tracking for non-linear teleoperators with variable time delay," Int. J. Robot. Res., vol. 28, pp. 895-910, 2009.

[4] P. F. Hokayem and M. W. Spong, "Bilateral teleoperation: An historical survey," Automatica, vol. 42, pp. 2035-2057, 2006.

[5] E. Nuño, L. Basañez, and R. Ortega, "Passivity-based control for bilateral teleoperation: A tutorial," Automatica, vol. 47, pp. 485-495, 2011.

[6] D. Sun, F. Naghdy, and H. Du, "Application of wave-variable control to bilateral teleoperation systems: A survey," Annu. Rev. Control, vol. 38, pp. 12-31, 2014.

[7] W. M. Bergmann Tiest, "Tactual perception of material properties," Vis. Res., vol. 50, no. 24, pp. 2775-2782, 2010.

[8] R. Harper and S. S. Stevens, "Subjective hardness of compliant materials," Quart. J. Experimental Psychol., vol. 16, no. 3, pp. 204215,1964

[9] F. Coppen, "The differential threshold for the subjective judgement of the elastic and plastic properties of soft bodies," Brit. J. Psychol. General Section, vol. 32, no. 3, pp. 231-247, 1942.

[10] G. W. S. Blair and F. M. V. Coppen, "The subjective judgment of the elastic and plastic properties of soft bodies: The "differential thresholds" for viscosities and compression moduli," J. Proc. Roy. Soc. Series B Biol. Sci., vol. 128, no. 850, pp. 109-125, Dec. 1939.

[11] D. Lawrence, L. Y. Pao, A. Dougherty, M. Salada, and Y. Pavlou, "Rate-hardness: A new performance metric for haptic interfaces," IEEE Trans. Robot. Autom., vol. 16, no. 4, pp. 357-371, 2000.

[12] G. Han and S. Choi, "Extended rate-hardness: A measure for perceived hardness," in Proc. Int. Conf. Haptics: Generating Perceiving Tangible Sensations, 2010, pp. 117-124.

[13] L. Rosenberg and B. Adelstein, "Perceptual decomposition of virtual haptic surfaces," in Proc. IEEE Symp. Res. Frontiers Virtual Real., 1993, pp. 46-53.

[14] F. E. Van Beek, D. J. F. Heck, H. Nijmeijer, W. M. Bergmann Tiest, and A. M. L. Kappers, "The effect of damping on the perception of hardness," in Proc. IEEE World Haptics Conf., 2015, pp. 82-87.

[15] S. Coren, The Left-Hander Syndrome. New York, NY, USA: Vintage Books, 1993.

[16] R. van der Linde and P. Lammertse, "HapticMaster-A generic force controlled robot for human interaction," Indus. Robot Int. J., vol. 30, no. 6, pp. 515-524, 2003.

[17] I. Nisky, F. Mussa-Ivaldi, and A. Karniel, "A regression and boundary-crossing-based model for the perception of delayed stiffness," IEEE Trans. Haptics, vol. 1, no. 2, pp. 73-82, Jul./Dec. 2008.
[18] M. D. Luca, B. Knörlein, M. Ernst, and M. Harders, "Effects of visual-haptic asynchronies and loading-unloading movements on compliance perception," Brain Res. Bulletin, vol. 85, no. 5, pp. 245$259,2011$.

[19] L. A. Jones and I. W. Hunter, "A perceptual analysis of stiffness," Experimental. Brain Res., vol. 79, no. 1, pp. 150-156, 1990.

[20] N. Sarkar and X. Yun, "Design of a continuous controller for contact transition task based on impulsive constraint analysis," in Proc. IEEE Int. Conf. Robot. Autom., 1996, pp. 2000-2005.

[21] K. Kuchenbecker, J. Fiene, and G. Niemeyer, "Improving contact realism through event-based haptic feedback," IEEE Trans. Vis. Comput. Graphics, vol. 12, no. 2, pp. 219-230, Mar. 2006.

[22] M. A. Srinivasan and R. H. LaMotte, "Tactual discrimination of softness," J., Neurophysiol., vol. 73, no. 1, pp. 88-101, 1995.

[23] W. M. Bergmann Tiest and A. M. L. Kappers, "Cues for haptic perception of compliance," IEEE Trans. Haptics, vol. 2, no. 4, pp. 189-199, Oct./Dec. 2009.

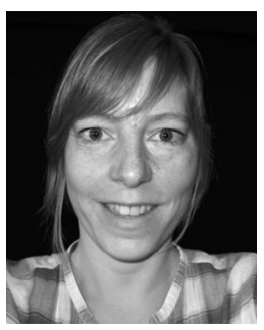

Femke E. van Beek received the MSc degree in biology from Wageningen University, the Netherlands, in 2010. After working as a data analyst for two years, she is currently working toward the $\mathrm{PhD}$ degree. She started as a PhD student in the Physics of Man Group at Utrecht University, within the Helmholtz Institute. As the group moved, she is currently working in the Department of Human Movement Sciences, Vrije Universiteit Amsterdam. Her research interests include human perception, haptics, and psychophysics.

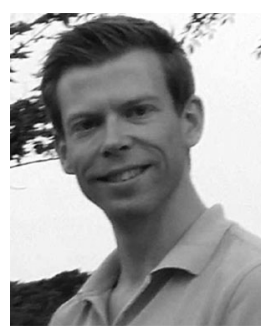

Dennis J.F. Heck received the BSc and MSc degrees in mechanical engineering at the Eindhoven University of Technology, the Netherlands. His master thesis research focussed on motion and force control for cooperative manipulation under the supervision of Prof. H. Nijmeijer. In 2015, he received the $\mathrm{PhD}$ degree at the Dynamics and Control Group, Eindhoven University of Technology, under the supervision of Prof. Dr. H. Nijmeijer and Dr. A. Saccon. His research, which was supported by STW and part of the $\mathrm{H}$-Haptics project, focused on the controller design for delayed bilateral teleoperation.

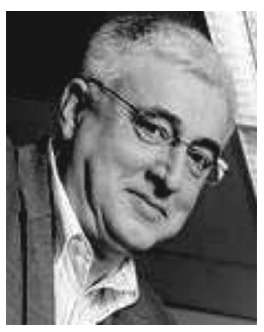

Henk Nijmeijer is currently a full professor at Eindhoven, and chairs the Dynamics and Control Group. He has published a large number of journal and conference papers, and several books, and is or was on the editorial board of numerous journals. $\mathrm{He}$ is an editor of Communications in Nonlinear Science and Numerical Simulations. $\mathrm{He}$ was appointed an honorary knight of the 'golden feedback loop' (NTNU) in 2011. Since 2011, he has been an IFAC Council Member. In January 2015, he became the scientific director of the Dutch Institute of Systems and Control. He received the 2015 IEEE Control Systems Technology Award. He has been a fellow of the IEEE since 2000 and was awarded in 1990 the IEE Heaviside Premium. 


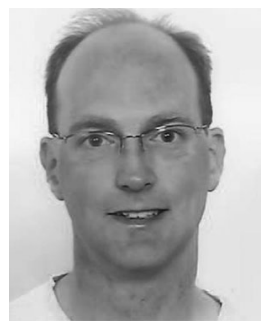

Wouter M. Bergmann Tiest received the MSc degree in experimental physics from Utrecht University, the Netherlands, in 1999. Until 2004, he was employed by the Netherlands Institute for Space Research, while getting the PhD degree from Utrecht University. After working as a postdoctoral in the Department of Physics and Astronomy, Utrecht University, in the Human Perception Group of the Helmholtz Institute, he is currently a researcher at the Department of Human Movement Sciences, Vrije Universiteit Amsterdam. His research interests include haptic perception of volume, mass, force, velocity, and material properties. $\mathrm{He}$ is an associate editor of the IEEE Transactions on Haptics.

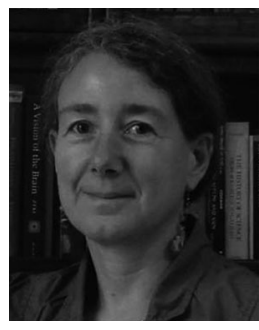

Astrid M.L. Kappers studied experimental physics at Utrecht University, the Netherlands. She received the $\mathrm{PhD}$ degree from Eindhoven University of Technology. From 1989 till September 2012, she was with the Department of Physics and Astronomy, Utrecht University. From 20082012, she was head of the Human Perception Group of the Helmholtz Institute. In September 2012, she moved with her whole group to the MOVE Research Institute, Department of Human Movement Sciences, Vrije Universiteit Amsterdam, the Netherlands. She was promoted to full professor in 2005. Her research interests include haptic and visual perception. In 2003, she won the prestigious $\mathrm{VICl}$ grant. She is/was member of the editorial boards of Acta Psychologica (2006-present) and Current Psychology Letters (2000-2011) and associate editor of the IEEE Transactions on Haptics (2007-2011).

$\triangleright$ For more information on this or any other computing topic, please visit our Digital Library at www.computer.org/publications/dlib. 